effects of firm pressure on different parts of the abdomen with regard to the sensations which it excites, the state of the skin, and, if the malady is of a chronic character, the temper and condition of the mental faculties. It should be particularly noticed whether the surface of the tongue is red, and of a granular or smooth appearance ; whether irritating and solid ingesta give rise to pain and distress in the stonach, or tormina in the bowels; whether pressure on any part of the abdonen gives rise to pain or a feeling of soreness ; whether the skin in connection with these symptoms be dry, harsh and contracted, and whether the temper is morose, gloomy, taciturn and irritable. The particular application of these phenomena will be fully illustrated in a subsequent part of this essay.-Western Medical Gazette.

\title{
APPLICATION OF CAOUTCHOUC TO SURGICAL PURPOSES.
}

BY DR. R. RICHARDSON, OF WELLGURG, va.

Among the many improvements introduced of late years into the practice of surgery, no one, perhaps, is more conspicuous, than that in relation to the treatment of wounds. Instead of cramming them with tents or syndons, and other irritating foreign bodies; instead of introducing into them stimulating balsams, or applying escharotics, as was formerly the custom, causing severe and protracted sufferings to the patient, and occasioning a large and unseemly scar, the simple and rational mode is now adopted of bringing the separated parts together, and uniting them by the first intention. How much does not the profession owe to the immortal Hunter, for those doctrines upon which is founded a practice so natural and so beneficial!

This mode of treatment having obtained in all cases of wounds, except a few attended by peculiar circumstances, and it being universally conceded, that if the sides of a wound be properly brought into contact, and carefully retained there, they will form the most perfect union, with the least possible deformity, it becomes important to decide what means are calculated to effect this object in the best manner. Two kinds of sutures have been chiefly employed for this purpose; one, formed by passing a needle armed with a ligature through the edges of the wound, called the "bloody suture" (sutura cruenta), because blood followed the needle ; and the other formed by two slips of adhesive plaister, appliet on each side of the wound, and in a line with it, each having a broad selvage, which being joined together by sewing with a common needle, constituted the "dry suture" (sutura sicca), a title which has been improperly bestowed by many upon the common application of adhesive strips, where no sewing is used. Latterly, however, every kind of suture has given place to strips of adhesive plaister, supported by compresses, and an uniting bandage, except in a few cases, as the harelip, \&c., in which experience has hitherto decided in favor of the twisted suture. And there can be no question but that the adhesive plaister has deservedly superseded the suture, and that it affords the best means yet devised of promoting the union of parts by the first intention. 
It occurred to me, however, some time ago, that a considerable improvement might be made in the manner of its application. I found it to be somewhat difficult, or indeed impossible, with the common adhesive strips, to retain accurately in contact the edges of those wounds by which the muscular fibre was divided transversely, or which occurred in parts where some degree of inotion could not be prevented. This was the case particularly in wounds of the face, where, too, it was very desirable that the most perfect union of the edges should be obtained, in order to obviate the deformity of an extensive scar. I found it difficult to effect this : 1st. Because, with a good assistant, which can seldom be obtained, to keep the edges of the wound together while you apply the strips, you cannot bring them sufficiently close. 2nd. Because the natural elasticity or contractility of the divided parts, or a slight degree of motion, occasioned a partial separation or retraction" of the edges, even when you at first succeeded in applying them closely to each other ; and $3 d$. Because the oozing from the wound, by penetrating and moistening the linen upon which the plaister is spread, rendered the strips less adhesive.

Considering the great obstacle to be the textural contractility of the parts divided, the thought suggested itself to me, that if, instead of linen, the adhesive plaister were spread upon some elastic substance, which would admit of being applied in a state of tension, so that its clasticity being made to act in a contrary direction, might thus oppose and countcract that of the divided parts, the difficulty would be removed.

The only substance which presented itself as capable of this applica-

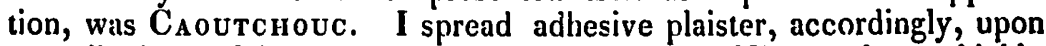
a small sheet of it, and made the experiment. The result was highly satisfactory, and the desired object completely attained. I found that it rendered the aid of an assistant entirely unnecessary, as by first laying down one end of the strip, then making the necessary extension immediately over the wound, and laying down the other end, the edges of the wound were drawn together with the greatest accuracy, and with a force so gentle, and so well applied, as not to occasion the least pain. In bringing the edges together with the fingers, we always give considerable pain, as we canuot act without making some degree of pressure upon the wounded parts. I found, also, that as the caoutchouc kept up a constant traction, the tendency of the edges to separate was continually counteracted, and motion could effect no displacement, while the plaister continued to adhere. I discovered, too, that it adhered much better, for the caoutchouc not being affected by the discharge or oozing from the wound, retained its situation, where the common strips would have become more or less loosened.

Upon the whole, from the trials I have made, I think this application of the mechanical properties of this singular article deserving of attention. When the lips of a wound are not kept perfectly in contact, that inosculation of the vessels necessary to union by the first intention, becomes more difficult; and although it is true that the effused blood becomes n connecting medium between them, and enables them to unite in this manner, unless too widely separated, yet the least degree of separation, amounting in fact to a loss of substance, requires a greater exertion 
of the powers of nature, and causes a larger cicatrix. It is, therefore, highly important that the edges of wounds should be brought together as accurately as possible, in endeavoring to unite them by the first intention. For this purpose, I am convinced the method here recommended will be found superior to any hitherto enployed, bringing the edges of the wound more perfectly together without pain, retaining them constantly in their proper situation, and effecting a cure without a scar.

I have only to add, that the caoutchouc is susceptible of being made into sheets of any required thickness or size, and is now, indeed, employed to cover tables.-Ibid.

EFFECTS OF TARTARIZED ANTIMONY IN DILATING THE OS UTERI.

BY JOHN LANGLEY, ESQ., SURGEON.

At three o'clock, P. M., of Friday the 21st ult., I was called to attend a young woman of plethoric habit and rigid fibre, 21 years of age, in labor with her first child. I was then informed she had been suffering severe pains during the day, which having increased in intensity and frequency, her attendants deemed it necessary to send for me. On visiting her, I found she had pains of such unequivocal character, occurring every five or six minutes, that induced me to press an examination per vaginam, believing, from their nature, the labor must have progressed to an advanced stage ; but, to my surprise, I found the os uteri with difficulty admitting my finger, extremely rigid and unyielding, the foetal head presenting, and pressing firmly upon it ; the pelvis sufficiently capacious; the bowels had been frequently and freely evacuated; but she experienced a most distressing inclination to pass urine, without the power. Accompanying this state, there was a hard and frequent pulse, dry tongue and hot skin. To relieve the insupportable suffering from obstructed micturition, with difficulty I drew off rather more than a pint of urine, which so far gave relief; still the apparent, and no doubt real, suffering of my patient was excessive, the pains assuming a more than ordinary violent character. I determined forthwith to bleed her freely, which proposition panic-struck her mother and self, both of whom positively and resolutely refused a compliance with my wishes, and in such a manner as rendered a perseverance unavailing, notwithstanding I described, in strong terms to both, the risk they incurred from such contumacious conduct. In the hope of inducing a relaxation of the contiguous parts, I threw into the rectum four injections of thin warm water gruel, at intervals of about an hour; still the same relentless rigidity of the os tincæ, and all the parts concerned in parturition. Reflecting upon the relaxation which supervenes upon the spontaneous vomiting which so frequently occurs, from sympathetic gastric irritation, during the last stage of uterine dilatation, I thought myself justified in anticipating, or rather imitating, nature in her operation, and decided upon giving small doses of tartarized antimony, which I did in quantities of one-sixth of a grain every twenty minutes in a little gruel, unconsciously to my patient. The second dose produced considerable nausea and gentle diaphoresis; and, upon again examining, I found the os uteri in a more favorable state. I gave a third 